\title{
Über einige primäre und sekundäre Regulationen in der Entwickelung der Echinodermen.
}

\author{
Von \\ Hans Driesch.
}

Mit Tafel VIII.

Eingegangen am 8. Jnni 1896.

In einer früheren Stndie ${ }^{1}$ ) habe ich die Begriffe der primären nnd der sekundären ontogenetischen Selbstregulation aufgestellt. Primäre Regulationen der Ontogenese nannte ich alle solchen Ausgleiche von kïnstlich gesetzten Störungen, welche durch die der deskriptiv-normalen Ontogenese angehörigen Geschehensarten, also durch Faktoren, die, weil sie auch unter deskriptiv-anormalen Bedingungen sich äußern, in tieferem Sinne normal zu nennen sind, bewerkstelligt werden, während ich den Namen sekundäre Regulation jedem Ausgleichsgeschehnis gab, das durch Vorgänge herbeigeführt wird, welche der normalen Ontogenese fremd sind, wie es z. B. die bei der Regeneration stattfindenden Processe sind.

Ich habe nun gelegentlich anderer Untersuchnngen, welche den Inbalt eines späteren Aufsatzes bilden werden, Formen von Regulationen beider Art in der Entwickelung von Asterias zur Beobachtung. bekommen, welche mir eines eingehenderen Verfolgens werth erschienen; den Erfolg meiner Arbeit lege ich hier vor, wobei gleich bemerkt sei, dass die eine Art der zu schildernden Regulationen, die primären nämlich, Berührungspunkte mit Dingen boten, die ich frïher $\left.{ }^{2}\right)$ schon einmal gelegentlich von Seeigellarven schilderte, wesshalb denn auch diese frïheren Beobachtungen nochmals vorgenommen und bei dieser Gelegenheit erweitert worden sind.

1) $\gg$ Die taktische Reizbarkeit der Mesenchymzellen von Echinus microtuberculatus. A. E. M. III. pag. $377 \mathrm{ff}$.

2) Entw. Stud. VIII. Mitth. Neapel. XI. pag. 226. 


\title{
I. Der Ausgleich der äulseren Form bei faltigen Echinodermenblastulis.
}

\author{
A. Asteriden.
}

Wenn man Eier von Asterias glacialis auf dem zweizelligen Stadium ziemlich stark schüttelt, so wird nur sehr selten die eine der beiden Zellen abgetödtet, vielmehr bleiben meist beide Zellen scheinbar in ungestörten Verhältnissen erhalten, die Vornahme der Operation hat aber doch eine bedentsame Folge und zwar diese:

Wenn die Eier abgefurcht sind, aber noch keinen epithelialen Habitus besitzen, so zeigt die Wandung der "Morula" von einer oder von zwei gegenüberliegenden Seiten einschneidende, oft sehr tiefe und sich, im Falle es zwei sind, nahezu berührende Falten, welche den Objekten das Aussehen von theilweisen Zwillingen verleihen. Solche Falten an der Morula finden sich nun zwar auch bei ungestört entwickelten Asteridenlarven (im Gegensatz zu denen von Echiniden) nicht selten, gleichen sich hier bei der Blastnlation aber stets ohne Rest aus; das ist nun zwar anch mit vielen Falten an den geschüttelten Objekten der Fall, aber doch nicht an allen, und mit der Übertragung der Falten auf die echte Blastula beginnt das eigentliche Interesse des Versuchs.

Obwohl ich es nicht sicher konstatirt habe, halte ich dafür, dass die Falten der Wandung in ihrer Richtung der ersten Furchungsebene entsprechen: es spricht dafür, dass erstens doch eben auf dem Zweizellenstadium die Operation stattfand und dass zweitens, sobald zwei Faltungen vorhanden sind, diese immer von gegenüberliegenden Orten ausgehen und stets eine Ebene bestimmen.

Die Figuren 1 und 2 stellen solche gefaltete Blastulae von Asterias glacialis dar. Wir wollen uns in diesem Absehnitt nun nicht mit dem seltsamen Einfluss beschäftigen, welchen die eine der Falten, sei sie allein oder eine von zweien, eventuell auf die Darmbildung haben kann, wir handeln hier nur von Ektodermdeformationen, welche aus Faltungen der Blastula hervorgehen.

Ist eine Ektodermdeformation an der Gastrula vorhanden, so liegt sie stets dem Darmorte gegenüber ${ }^{1}$ ) (Fig. 3a), was auch dafür

1) Ich möchte an dieser Stelle auf einen Passus in 0. HeRTwig's »Präformation oder Epigenese? Bezug nehmen, der eine - übrigens ziemlich verbreitete - Ansicht vertritt, welche ich nicht theile und die mir durch die hier geschilderten Befunde direkt widerlegt zı werden scheint. Es heißt daselbst pag. 95: »... dadurch, dass in Folge der Einstülpung, welche aus den Wachs- 
spricht, dass die Falten der ersten Furche örtlich entsprechen, welche normalerweise wohl durch die Açse des als polarig anzunehmenden Eies geht.

Es ist nun eine beachtenswerthe Thatsache, dass Ektodermfalten, welche im Beginn der Gastrulation noch sehr tief gingen, bei vollendeter Gastrulation meist schon etwas seichter, zur Zeit der Mesenchymbildung aber meist völlig verschwunden sind, wenigstens sobald ihre Träger ein gesundes, munteres Verhalten zeigen. Die Figuren $3 b$ und $c$ sowie $5 a$ und $b, 14 a$ und $b$ zeigen den successiven Ausgleich der Faltung an mehreren Objekten.

Welche Vorgänge mögen ihm zu Grunde liegen?

Von vorn herein will ich die Anschaung zurïckweisen, dass etwa der wachsende Darm die Falte herausdrücke, eine Vorstellung, die schon desshalb gar keiner ernsthaften Diskussion werth ist, da bei Asteriden der Darm bekanntlich nicht durch die ganze Länge der Gastrula hindurch reicht, sondern in etwa $2 / 3$ der Achsenlänge frei endigt: er kommt mit einer Falte am animalen Pol also in der Mehrzahl der Fälle gar nicht in Berihhrung.

Wenn wir zu einem Verständnis des Faltungsausgleichs gelangen wollen, müssen wir uns, denke ich, dessen erinnern, was wir uber das Wachsthum der Asteridenlarve wissen. Da habe ich denn früher $\left.{ }^{1}\right)$ schon darauf hingewiesen, dass dieselbe zur Zeit der Mesenchymbildung ihr stärkstes Wachsthum zeigt, indem ihr Längsdurchmesser sich

thumsverhältnissen der Blasenwand zu erklären ist, eine Zellenfläche in neue Lagebeziehungen zu ihrer Umgebung gebracht wird, wird sie Entoderm, erhält sie den Anstoß, die ihrer besonderen Lage entsprechenden Eigenschaften zu entfalten. Ich sage nun zwar nicht, was HeRTwig mit seiner Anschaung kontrastirt, dass Zellen, „weil sie eine besondere Determinantengruppe besitzen ", za Entodermzellen werden, aber ich lasse, wie jede primäre Organbildung, so auch die Entodermbildung durch die präformirte Organisation des Eiplasmas ansgelöst werden, sehe also in einer inneren Induktion den Anstoß zu ihr, aber nicht in irgend welcher Veränderung der Beziehungen von Keimestheilen zur Außenwelt. Sind doch bei meinen gefalteten Blastnlis gerade durch die Faltung Wandungstheile des Keimes in abnorme Beziehungen zur Außenwelt, nämlich ins Innere des Blastocöls, gebracht worden: diese Theile werden aber durchans nicht zi1 Entoderm, sondern meist entsteht dieses gerade an solchen Keimorten, welche durchaus ihre normalen äußeren Beziehungen wahrten; das beweist, denke ich, dass von der uns leider nicht sichtbaren Orientirung der Keimestheile der Anstoß (die Induktion) zur Entodermbildung am bestimmten Orte ansgeht. Vgl. auch Anal. Theor. pag. 176, ferner »Organzellen.» A. E. M. II. pag. 196 und „Organisat. d. Eies." A. E. M. IV. 1) "Organzellen." A. E. M. II. pag. 178; siehe auch HerBst, Exp. Unt. IV. A. E. M. II. pag. 4 i8. 
dann um etwa die Hälfte verlängert; zugleich wird- dàs ektodermale Epithel diunner und zeigt seine Kerne weniger dicht liegend, so dass wir das geschilderte Wachsthum nicht als durch Theilung und Wachsthum jeder einzelnen Epithelzelle vermittelt ansehen dürfen, sondern einem vom Blastocöl ausgehenden dehnenden (osmotischen) Drucke zuschreiben müssen.

Ich bin nun geneigt, auch den Ausgleich ektodermaler Falten der Wirkung osmotischen Dehndruckes zuzuschreiben, und zwar vorwiegend desswegen, weil, wie wir sahen, der völlige Ansgleich der Falte sich stets zur Zeit der Mesenchymbildung vollzieht, zu einer Zeit also, in welcher, wie eben erörtert, auch das durch Dehnung vermittelte starke Wachsthum der Larve bei ungestörter Entwickelung statthat.

Ein Faktor, welcher in der deskriptiv-normalen Entwickelung des Asterias eine wichtige Rolle spielt, würde nach unserer Auffassung. also gleichzeitig zum Ausgleich von Formstörungen verwandt werden; der Ausgleich dieser Störungen wäre als ein in tieferem Sinne "normales " Geschehnis zu bezeichnen, nicht als ein besondere, neue Faktoren benöthigendes; eben darum wäre der Ausgleich von Störungen in der Konfiguration des Ektoderms von Asterias eine primäre Selbstregulation zu nennen. -

Es kommt bisweilen vor, dass die Darmanlage nicht, was wir als das Normale schilderten, der Ektodermbucht gegenüber liegt, sondern dass zwei Ektodermbuchten einander vis-à-vis gelegen sind und der Darm ganz der einen der beiden so gebildeten Blasen angehört (Fig. 4); diese seltsame Lage des Darmes diskutiren wir später, hier interessirt uns nur die Thatsache, dass auch in solchen Fällen sehr starker Ektodermdeformation ein völliger Ausgleich statthaben und eine durchaus glatte und normal gestaltete Larve zur Zeit der Mesenchymbildung resultiren kann (Fig. $5 a$ und $b$ ), ein Vorkommnis, welches auf einen starken Energiewerth des von uns zum Ausgleich in Anspruch genommenen osmotischen Druckes im Blastocöl hinweist.

\section{B. Echiniden.}

Im Jahre 1893 schilderte ich es anlässlich meiner Versuche iiber den Einfluss verdünnten Seewassers auf die Furchung kurz, dass Blastulae von Echinus microtuberculatus, welche sich in Seewasser eutwickelt haben, dem $30 \%$ Brunnenwasser zugesetzt war, zum großen Theil eine seltsam gestreckte, biskuitförmige Gestalt 
darbieten, dass sie jedoch, in reines Seewasser zurïckgebracht, oder auch in solches, dem nur etwa 15\% Brunnenwasser zugesetzt wurden, sich normal zu entwickeln und auch starke Ektodermbuchten auszugleichen im Stande sind.

Ich habe diese Beobachtungen noch einmal geprifft und vollauf bestätigt gefunden.

Die Fig. I zeigt eine $e_{4}^{8}$ solche in verdünntem Seewasser biskuitförmig ansgebildete Blastula von Echinus im Umriss; die Gebilde erwecken den Eindruck, als wollten sie Zwillinge bilden, wie denn ja in der That seiner Zeit häufig die Bildung zweier kleiner Larven aus einem Ei in verdünntem Seewasser zur Beobachtung gelangte. Gleichwohl entwickeln sich unsere nur gezerrten nicht getrennten Larven in der großen Mehrzahl der Fälle als e in Ganzes. Wie Fig. I zeigt, liegt der Bildungsort des Mesenchyms und Darmes in der ringförmigen Senkung, welche die beiden Blasen des bisknitförmigen Körpers trennt; Fig. II zeigt deutlich, wie um ihn herum die Mesenchymzellen in kreuzförmiger Anordnung und in normaler') Zahl von etwa 60 gelegen sind.

Diese Lage des Darmbildungsortes spricht dafür, dass die ringförmige Furche der ersten Furchungsebene des Eies, welche durch seine Achse geht, entspricht, wie ja auch im Wärmeofen ${ }^{2}$ ) Blastulae stets in Richtnng der ersten Furche gezerrt und in ihr eventuell zur Zwillingsbildung veranlasst werden, nie aber in anderen, Thatsachen, für die wir zur Zeit keinen Grund anzugeben vermögen.

Die ringförmige Bucht des Ektoderms reducirt sich nun meist schon im Verlauf des Darmwachsens auf eine ḳleine dem vegetativen Pol gegenüberliegende Senkung (Fig. III) und mit vollendeter Darmbildung ist meist jeder Rest derselben verschwunden (Fig. IV), während gleichzeitig die bei Echinns so frih ausgeprägte bilaterale Prismenform der Larve mit ihrer seitlichen Lage des Urdarmes sich ausbildet; der Urdarm legt sich in diesem Stadium mit seinem freien Ende der stärker gewölbten Wandungsfäche der Prismenlarve an; später entsteht auf der ebenen Wandungsfläche derselben der Mund, der Darm setzt sich durch eine Schwenkung ${ }^{3}$ ) mit ihm in Verbindung, gliedert sich in Vorder-, Mittel- und Enddarm und die Pluteusausbildung ist nahezu fertig: alle diese Vorgänge geschehen

\footnotetext{
1) Vgl. »Takt. Reizb.« A. E. M. III. pag. 366.

2) Entw. Stud. IV a. Zeitschr. f. wiss. Zool. LV. pag. $10 \mathrm{ff.}$

3) Vgl. »Takt. Reizb.« A. E. M. III. pag. 369 Anm.
} 
an der großen Mehrzahl der Versuchsobjekte durchaus als sei ihnen nichts im Beginn ihrer Entwickelung geschehen.

Mit vollendeter Gastrulation, sicherlich wenigstens mit äußerlich (nicht nur in der Anordnung des Mesenchyms) ausgesprochener Bilateralität ist also das gebuchtete Ektoderm der Echinuslarven in seiner Form ausgeglichen. Fragen wir nach den nächsten Ursachen dieses Ausgleichs, so werden wir wieder, wie oben, ein Hinausdrängen der Ektodermfalte durch den wachsenden Darm ablehnen müssen, da der Ausgleich ja stets beginnt, oft sogar schon vollendet ist, ehe der Darm die gegenüberliegende Wandung irgendwie berïhrt: wir werden auch hier den im Inneren des Blastocöls herrschenden osmotischen Druck, welcher im normalen Wachsthum der Echinidenlarve mit Sicherheit eine Rolle spielt ${ }^{1}$ ), als ansgleichenden Faktor heranziehen, mag er hier auch nicht wie bei Asteriden in einem bestimmten Zeitmoment eine so starke plötzliche Steigerung erfahren.

Wir rechnen also auch bei Echinus die Ausgleichserscheinungen von Ektodermfalten zur Klasse der primären Regulationen ${ }^{2}$.

Obwohl Alles mit der Frage nach der Darmentstehung der Zerrlarven im Theil II dieses Aufsatzes erörtert werden soll, müssen wir hier doch mit einigen Worten auf das Verhaiten des Darmes nach seiner Anlegung eingehen.

Zunächst wäre Einiges über die Richtung seịnes Wachsens zu sagen, freilich nur dieses, dass wir etwas Wesentliches über sie nicht zu sagen wissen; sie diurfte von der Orientirung' der vegetativen Platte, welche nach Morgas ${ }^{3}$ ja die Darmbildung der Echiniden einleitet, abhängen, indem sie immer senkrecht auf dicser steht; die Platte hinwiederum hängt örtlich von der Lage des vegetativen Poles des Eies und seiner nächsten Umgebung ab, welche sich auf den abgefurchten Keim und die Blastula uibertragen. Liegt also wie meist die in der Ringbucht befindliche Darmplatte durchans symmetrisch zum Ganzen (Fig. I), so folgt daraus auch eine symmetrische Wachsthumsrichtung des Darmes, sonst eine irgend wie zum Ganzen geneigte; und zwar sieht man ein, dass geringe Abweichung von der

1) Herbst, Exp. Unters. II. Mitth. Neapel XI. pag. 148 und sonst; siehe auch meine Oryanzellen. A. E. M. II. pag. 175.

2) Wir handeln hier vom Ausgleich deformirter Blastulae, nicht vou der Entstehung ihrer Deformation, die deformirte Blastula nehmen wir als gegeben hin; die Arbeit Loes's, Beiträge zur Entwickelungsmechanik der aus einem Ei entstehenden Doppelbildungen. A. E. M. I. pag. 453 ff. berïhrt also unser Problem nicht näher und es genïgt, dieselbe hier genannt zu haben.

3) p Partial Larvae.« A. E. M. II. pag. $84 \mathrm{ff}$. 
Symmetrie [Fig. VIII) bei der Platte eine weit stärker sichtbare am vollendeten Darme nach sich ziehen muss (Fig. V $a$, VII $a$, VIII $b$ ).

Sehr bald werden ja übrigens die primären Lageverhältnisse des Darmes der Echiniden zum Keimganzen wesentlich geändert, und zwar nicht durch Vorgänge an ihm selbst, sondern durch Ausbildung der oben erwähnten bilateralen Prismenform des Ektoderms.

Wie oben bemerkt waren die Ausbildnng dieser Prismenform, die Lage des Darmes in ihr und seine spätere Wendung zum Munde hin bei unseren Objekten in der großen Mehrzahl der Fälle ganz oder fast normal, und das gilt nun auch von Larven, deren Darm Anfangs recht seitliche Lage zeigte (Fig. V $a$ und $b$ ); durch die Regulation der Form des Ektoderms ist hier also die relative Lage des Darmes im Keimganzen zugleich mit regulirt worden. Bisweilen erfolgte die Wendung des freien Darmendes von der stark gekrümmten Wandung des Ektoderms der Prismenlarve fort zu ihrer ebenen Fläche hin auffallend spät, indem auf letaterer der Mund bereits deutlich ansgebildet war, während der Darm noch weit von ihm entfernt an der gekrümmten Wandungsseite endigte (Fig. VI); am nächsten Tage hatte freilich auch dieser Darm die Wendung richtig vollführt und sich zugleich in seine drei Theile gegliedert; es entstand ein normaler Pluteus.

Man könnte geneigt sein, in dieser Wendung des Darmes zum Mund hin eine tropische Erscheinung, einen Richtungsreiz ${ }^{1}$ ), zu erblicken, freilich mag das örtliche Zusammenpassen von Mund und Darm auch ohne intimere Kausalverkettung lediglich auf Grund der Kompositionsharmonie ${ }^{2}$ ) des Keimganzen zu Stande kommen.

Ein interessanter Fall liegt mir vor, in welehem das Zusammentreffen von Mund und Darm nicht erfolgte, letzterer vielmehr um $90^{\circ}$ verkehrt liegen blieb und sich in dieser falschen Lage in seine drei Theile gliederte, so dass der im Übrigen normale Pluteus sehr seltsam gestaltet war (Fig. VII $b$ ). Es war dieses Gebilde aus einer Gastrula hervorgegangen, deren Darm von Anfang an recht unsymmetrisch gewachsen war (Fig. VII $a$ ) und nicht erst als Prismenform, wo das, wie gesagt, normal ist, sehr seitliche Darmlage zeigte; eine etwas asymmetrische Orientirung der Darmplatte in der Ringbucht haben wir nach dem oben Gesagten wohl als Ursache dieses Verhaltens anzusprechen.

1) ef. Herbst, »Richtungsreize. « Biol. Centralbl. XIV. pag. $771 \mathrm{ff}$.

9) Anal. Theorie pag. 130. 
Warum hier nun freilich die definitive Darmwendung nicht erfolgte, ist damit noch nicht gesagt; erfolgte sie doch in zahlreichen ähnlichen Fällen in typischer Weise. Ich bin geneigt hier eine aus irgend welchen Gründen eingetretene Verlöthung des freien Darmendes mit derjenigen Ektodermstelle, an die es gerade im Wachsen traf, anzunehmen, eine Verlöthung; welche auch sonst wohl bisweilen vorkommt, hier aber so intensiv war, dass die gut eintretende Formregulirung des Ektoderms und seine spätere Prismenformausbildung nicht, wie sonst, Richtungsabnormitäten des Darmes mit zu reguliren im Stande war, sondern ihn in seiner abnormen Situation beließ, wo er dann sich weiter differenzirte. Übrigens ein gutes neues Beispiel für die Unabhängigkeit der Entwickelung von Ektoderm und Entoderm, für ihre „Selbstdifferenzirung «") in Bezug auf einander.

\section{Die Darmentwickelung von deformirten Echinodermenblastulis und ihre Regulationen.}

Wir gehen dazu über, die merkwürdigen Vorgänge zu schildern, welche die durch Schütteln oder Anfenthalt in verdünntem Seewasser deformirten Blastulae von Asteriden und Echiniden hinsichtlich ihrer Darmentwicklung in vielen Fällen darbieten. Um vom Einfachen zum Komplicirteren vorzuschreiten, beginnen wir mit den

\section{A. Echiniden.}

\section{Primäre Regulationen.}

Wir sahen, dass an Blastulis mit ringförmiger Einbuchtung die Darmplatte sich in der großen Mebrzahl der Fälle an einer Stelle der Bucht, und zwar meist durchaus symmetrisch zum Ganzen anlegt, und dass das Mesenchym in normaler Zellenzahl (50 bis 60) kreisförmig mit erkennbarer Bilateralität um diese Platte herum angeordnet ist (Fig. I und II); der Keim bildet ein freilich deformirtes Ganzes. Auch in einigen Füllen, wo der Darm etwas seitlich und zum Ganzen unsymmetrisch gewachsen war (Fig. Va), glanbten wir den Keim als ein Ganzes ansprechen und in diesem Verhalten nur die stärker sichtbare Folge einer geringen Asymmetrie der Darmplatte sehen zu dürfen; entwickelten sich doch solche Gebilde in der That zu einem ganzen Pluteus.

Ich habe nun schon früher beobachtet und oben wieder daraud

1) Anal. Theorie. pag. $92 \mathrm{ff}$. 
Primäre a. sekundüre Regulationen in der Entwickelung der Echinodermen. 255

hingewiesen, dass andererseits aus Eiern, welche in verdünntem Seewasser geweilt haben, oftmals Zwillinge hervorgehen, d. h. zwei sich als Blastulae bereits trennende Individuen, deren jedes sich zu einem kleinen ganzen. Pluteus ausbilden kann. Hier sind aus einem Ganzen also offenbar zwei kleine Ganze geworden, und ich habe schon früher ${ }^{1}$ ) und kürzlich ${ }^{2}$ ) wieder ausführlich dargelegt, welche Arten von Vorgängen meines Erachtens bei der Bildung solcher nener verkleinerter Ganzen anzunehmen sind, Erwägungen, die indess nicht die Beachtung gefunden haben, die der Gegenstand, denke ich, erheischt, mögen sie selbst auch im Einzelnen ganz oder theilweise falsch sein.

Jetzt habe ich nun einige Geschehnisse beobachtet, welche mir auf diese Vorgänge, welche bei Bildung eines verkleinerten Ganzen in Frage kommen, einiges Licht zu werfen geeignet sind: es handelt sich um einige Gebilde, die als theilweise $\mathrm{Zwillinge} z$ bezeichnen sind.

Ich schicke der weiteren Schilderung die Bemerkung voraus, dass nach MoR $\mathrm{AAN}^{3}{ }^{3}$ ) die Zahl der Darmzellen in aus einer der ersten Blastomeren gezuichteten Theillarven nahezu ebenso viel wie diejenige normaler Larven beträgt, also relativ zu viel, während ich mich überzeugt habe, dass die Zahl der Mesenchymzellen bei solchen Gebilden im Stadium des vollendeten Ringes ${ }^{4}$ ) zwisehen 35 und 40 beträgt, was etwa $2 / 3$ der an normalen Larven beobachteten Zellenzahl (50 bis 60$)$ ausmacht.

Das erste der nunmehr zu schildernden Objekte war aus einer stark gebuchteten Blastula hervorgegangen, deren Darmplatte etwas unsymmetrisch zum Ganzen orientirt war (Fig. VIII $a$ ); nach einiger Zeit bemerkte man, dass Mesenchym ringförmig in beiden durch die starke Ringfurche getrennten Blasen vorhanden sei, Einzelheiten konnten an der munter schwimmenden Larve leider nicht konstatirt werden; am nächsten Morgen fand sich in einer der Blasen ein Darm, in der anderen immer noch wohl getrennten nicht (Fig. VIII $b$ ), und noch einen Tag später fand sich neben dem erwähnten wohl entwickelten Darm der einen Blase die Anlage zweier schon recht großer Skelettdreistrahler und in der anderen immer noch dureh die Bucht getrennten Blase fand sich zwar kein Darm, aber

1) Anal. Theor. pag. $19 \mathrm{ff}$.

2) $\rightarrow$ Organisat. d. Eies. A. E. M. IV. pag. 88.

3) "Partial Larvae." A. E. M. II. pag. $98 \mathrm{ff}$.

4) Vgl. »Takt. Reizb.« A. E. M. pag. $366 \mathrm{ff}$. 
auch die Anlage zweier großen Dreistrahler vor. Auf diesem Stadium wurde das Gebilde getödtet und gęnau gezeichnet (Fig. VIII $c$ ).

Es hat hier also nicht nur keine Regulation der ektodermalen Bucht stattgefunden, sondern es haben sich sogar die durch sie getrennten Blasen selbständig entwickelt, es ist ein Gebilde entstanden, welches in Bezug auf das Skelet ein $Z$ willing, und $z w a r$ ein verwachsener $Z$ willing ist, wozu noch kommt, dass jedes der beiden Theilindividuen die Prismenform deutlich angenommen hatte ${ }^{1}$.

Wie sollen wir diese Vorgänge deuten?

Erwägen wir zunächst einmal die Thatsache, dass, wie oben geschildert, die in Fig. $\mathrm{V} a$ dargestellte Zerrgastrula nicht etwa einen theilweisen Zwilling, sondern, nach vollzogenem Ektodermausgleich, welcher zugleich die relative Darmlage mit regulirte (Fig. $\mathrm{V} b$ ), einen annähernd normalen Pluteus producirt hat: hiet sind also nicht zwei Ganze aus einem Ganzen geworden, trotz großer Ähnlichkeit der Ausgangsform (man vergleiche die Figuren V $a$ und VIII $b$ ); der Darm dieser Larve ist offenbar dem Werthe nach der Darm einer Ganzlarve; entspricht nun wohl der eine Darm, welchen unser Zwilling nur besitzt, dem Darm einer Ganzlarve oder entspricht er dem einer Theillarve und ist, wie das ja bisweilen bei Theillarven vorkommt, die Darmbildung in der darmlosen Blase nur' unterdrückt worden? Mit anderen Worten: ist unser theilweiser Zwilling wirklich dem einen Organsystem, dem Darm nach ein Ganzes, dem anderen, dem Mesenchym nach, zwei Ganze, oder stellt er zwei Ganze dar, in deren einem eine Bildung unterdriickt ist? Man könnte aus der Thatsache, dass in der normalen Echinidenentiwicklung Darm- und Mesenchymbildung so eng zusammenhängen, ohne Weiteres die zweite Alternative zu folgern geneigt sein; ich denke aber, das geht doch nicht so ohne Weiteres, und zwar aus zwei Gründen:

einmal war bei unserem Objekt, wie die Skizze Fig. VIII $a$ zeigt,

1) LoEB hat in seiner oben erwähnten Arbeit auch einiger Fälle von Doppelskeletbildung an im Übrigen einfachen Larven Erwähnung gethan, die Genese dieser Gebilde jedoch nicht an einem und demselben Exemplar successiv verfolgt. Ist doch, wie schon bemerkt, sein Hauptproblem die Frage, wie Spaltung des Keimes entstehe, während die beiden sich dieser anschließenden Fragen, wie im Spaltprodukt oder im theilweise gespaltenen Keim die Rekonstruktion des Ganzen im verkleinerten Maßstabe erfolge und wie, in anderen Fïllen, ein theilweises Spaltprodukt sich wieder zum Ganzen im ursprïnglichen Maßstabe ausgleiche, außerhalb seiner Problemsphäre liegen, wogegen sie gerade die unsrige bilden. 
die vegetative Platte einheitlich in der Bucht, wennschon nicht ganz symmetrisch, angelegt;

zum Anderen ist unser Gebilde ja ein Zwilling, nicht in Rücksicht auf die Bildung, sondern in Rücksicht auf die Ordnung des Mesenchyms; vou dieser habe ich aber nachgewiesen ${ }^{1}$ ), dass sie durch attraktive Zonen im Ektoderm auf Grund taktischer Reizbarkeit der Mesenchymzellen erfolgt, so dass sie eigentlich ein ektodermales $P h \ddot{a n o m e n ~ i s t . ~}$

Ich halte also daftir, dass unser Gebilde dem Entoderm nach ein Ganzes, dem Ektoderm nach zwei Ganze repräsentirt, und zwar letzteres desshalb, weil erstens zweimal die Prismenform ausgebildet und zweitens zweimal die attraktiven Ringzonen vorhanden sind, letzterer Umstand zieht die Doppelheit des Mesenchyms unmittelbar nach sich, und daraus folgt wieder die Doppelheit des Skelets.

Dass der Darm ganz und gar einem der zwei Ektodermganzen angehört, wtirde ein gleichsam zufälliges Faktum sein: er blieb dort, wo er einmal war, da ja ein sonst auch ihn in der relativen Lage zum Ganzen regulirender Formausgleich, nämlich derjenige des Ektoderms, ausblieb.

Hiermit sind wir nun zu der Kardinalfrage gelangt: hat die Bildung zweier Ektodermganzer den Ausbleib der Formregulation oder hat letzterer die Bildung ersterer zur Folge?

Gerade auf Grund jener Larve, welche im Anfangsstadium unserem Zwilling so ähnlich war (Fig. Va), und sich dann doch regulirte und ein Ganzes bildete (Fig. Vb), möchte ich hier der zweiten Alternative zuneigen: ich denke mir also, dass unser Objekt zur Zeit der Anlage von Mesenchym und Darm ein Ganzes repräsentirte, indem das Ganze seiner Eiorganisation 2) zwar deformirt aber doch nicht in seiner Polarität wesentlich gestört war; dann aber, so nehme ich an, waren für den Ausgleich der vorhandenen starken Falten durch die Wirkung des osmotischen Druckes im Blastocöl zu große Widerstände vorhanden, so dass dieser Ausgleich unterblieb. Eben darin, also im Erhaltenbleiben der zweiblasigen Konfiguration ${ }^{3}$, liegt dann weiter der Anstoß dafür, dass das primäre Ganze der vom Eiban überkommenen Ordnung der Theilchen sich zu zwei neuen kleinen Ganzen ordnet, und zwar kann nun diese Neuordnung zu zwei Ganzen einen formativen Einfluss nur noch auf

1) sTakt. Reizb.« A. E. M. III.

2) 》0rganisat. d. Eies. A A. E. M. IV.

3) Ebenda. A. E. M. IV. pag. 88. 
alle die Elementarprocesse ausüben, welche noch nicht abgelaufen sind: es bildet sich also nicht etwa nochmals (und zwar zweimal) Darm und Mesenchym, aber die Ordnung des vorhandenen Mesenchyms geschieht zweifach, da eben zwei kleine ganze attraktive Ektodermzonen entstanden sind.

Ich weiß selbst am besten, wie viel Problematisches dieser Auffassung anhaftet und würde sehr erfreut sein, wenn man mir eine bessere böte; meine Auffassung des Darmes unseres Objektes, von der alles Andere abhängt, als einer dem Großganzen, nicht einem Kleinganzen zugehörigen Bildung halte ich allerdings für ziemlich gesichert. -

Es liegen mir noch zwei dem Geschilderten ähnliche Objekte vor; zunächst eines, das dem beschriebenen im Wesentlichen ähnlich war und daher keine besondere Diskussion erheischt, es mag nur erwähnt sein, dass hier die beiden Blasen, die darmlose und die darmhaltige, nur durch einen sehr engen Blastocölkanal verbunden waren. Bei dem zweiten Objekte kam überhaupt kein Darm zur Ausbildung, es starb nach zwei Tagen ab, dagegen konnte man deutlich die einheitliche Entstehung des Mesenchyms und seine dem ungeachtet doppelte Ordnung in zwei Ringen (Fig. IX) erkennen; auch hier war fast jeder Ektodermausgleich unterblieben.

In dem Umstande, dass ein-Ei sich theilweise als ein Ganzes, theilweise als zwei Ganze entwickeln kann, scheint mir das eigentliche Interesse dieser und der im Folgenden noch zu schildernden Thatsachen zu liegen. Die von anderen'Formen bekannten y) partiären Doppelbildungen lehren ja freilich Ähnliches, gestatten aber keine so gute Analyse. Auch meine Objekte sind nicht so gut analysirt, wie das an und für sich möglich wäre, man muss aber erwägen, dass ihrer unter 62 isolirt studirten Zerrblastulae von Echinus nur drei waren, und davon ein kränkliches; ferner ist in Betracht zu ziehen, dass man es, wie erwähnt, einer Zerrblastula im Beginn der Gastrulation nicht ansieht, was aus ihr wird, dass man daher auf später interessante Objekte nicht von Anfang an die Sorgfalt verwendet, die gerade sie erheischen und endlich - dass alle Beobachtungen mit Ausnahme der letzten an rasch schwimmenden Larven in relativ großen Gefäßen zu machen sind, was ein minutiöses Studium natürlich verbietet.

1) Man vergleiche zumal in WILson's "Amphioxus", Journ. of Morph. VIII. die Figuren $64-67$. 
Endlich mag noch erwähnt sein, dass der im Theil I meiner "Entwicklungsmechanișchen Studien "1) geschilderte und abgebildete Verwachsungszwilling etwas principiell Anderes als die hier geschilderten Objekte darstellt, denn bei ihm geschahen alle Organbildungsprocesse ron Anfang an doppelt, er luatte zwei Mesenchymentstehnngsorte, zwei Därme, kurz er repräsentirte von Anfang an zwei Theilblastulae, zwei kleine Ganze, die nur nicht, wie sonst, getrennt, sondern in kleinem Bezirk verwachsen waren.

\section{B. Asteriden.}

\section{a. Primäre Regulationen.}

Auch die aus geschüttelten $Z_{w}$ eizellenstadien hervorgegangenen Zerrblastulae von Asterias bilden bisweilen Gastrulae, deren Darm seitlich in einer Blase gelegen (Fig. 4, 5a), und es entsteht nun anch hier die Frage: haben wir in solchem seitlichen Darm den Darm einer von zwei Tlieillarven vor uns, während der Darm in ihrem Genossen nnterdrückt ist, oder liegt uns ein Ganzdarm in seitlicher Lage vor?

Hinsichtlich der ähnlichen Bildungeu von Echiniden hatten wir uns für die zweite Alternative entschieden und zwar vorwiegend desswegen, weil die Añlage des Darmes, die Darmplatte, einheitlich und stets im Grunde der Bucht, wennschon nicht - eben das bedingte, wie wir dachten, die seitliche Lage - ganz symmetrisch zum Ganzen gelegen war.

Bei den ähulichen Asteriaslarven füllt nun dieser Grund für Annahme der zweiten Alternative hinweg: hier entsteht der seitliche Darm deutlich seitlich in einer der beiden Blasen, neben einer der beiden Buchten, wie Fig. $5 a$ besouders dentlich zeigt. Da nun ferner mit ziemlicher Sicherheit anzunehmen ist, dass die Buchten der ersten Furchungsebene entsprechen, weil doch eben auf dem Zweizellenstadium geschüttelt war, und da die erste Furche wohl durch animalen und vegetativen Pol des Ganzen geht, so möchte ich furr die Asteridenlarren mit seitlichem Darm die Deutung vertreten, dass bier nicht der vegetative Pol des Eiganzen verlagert, sondern dass hier zwei neue vegetative Pole durch Umordnung der Keimesorganisation zu zwei neuen Ganzen von Anfang an entstanden seien, an derem einen zwar eine ontogenetische Folge, die Darmbildung. unterdruickt ist. Ich gebe also diese, von der für die scheinbar gleichen Verhältnisse an Echiniden abweichende Deutung, weil mir für

1) Zeitschr. f. wiss. Zool. LIII. pag. 174. 
die seitliche Lage des Darmbildungortes sonst der zureichende Grund fehlt.

Gestiitzt wird nun, denke ich, diese meine Anffassung durch das Vorkommen von Larven, deren Ektoderm eine den Fig. 4 und $5 a$ entsprechende Buchtung besitzt, und die in jeder Blase einen typischen Darm anlegten; eben darin, dass auch bei diesen Gebilden mit zwei Därmen die Ektodermbucht durchans nicht etwa zur völligen Trennung der Blasen geführt hatte, schließe ich, dass auch bei solchen Larven, welche nur einen, aber seitlichen Darm besitzen, die Bedingungen zur Konstruirung zweier potentieller »Ganze« gegeben gewesen seien.

Das eine der Gebilde mit zwei weit von einander getrennten Därmen ist in Fig. 6 dargestellt, es starb leider bald, ohne sich weiter entwickelt $z \mathfrak{u}$ haben; das andere ist von höchstem Interesse, und zwar vorwiegend desshalb, weil hier die Därme nicht gleichsinnig zu einander orientirt waren. Der eine Darm stand nicht nur zu dem anderen ungefähr senkrecht, sondern lag auch nicht einmal mit ihm in gleicher Ebene, was auf der Skizae (Fig. $7 a$ ) leider nur unvollkommen zum Ausdruck gelangt.

Ich führe dieses seltsame Vorkommen auf eine durch das Schiitteln verursachte Drehung der beiden ersten Blastomeren gegen einander zurück, so.dass also beider vegetative Abschnitte von einander getrennt wurden, ähnlich wie das für die ungleichsinnig orientirten Zwillinge, welche WILson ${ }^{1}$ ) von Amphioxus beschrieben hat, anzunehmen ist.

Um nun zunächst die Schilderung unserer merkwürdigen Zwillinge zu beenden, so sei bemerkt, dass am nächsten Tage der eine Darm blasige Auftreibung seines Endes zeigte, während der andere bereits in Cölombildung begriffen war, sowie dass beide Mesenchym mit normaler Vertheilung producirt hatten (Fig. 7b).

Noch einen Tag später waren je zwei Cölomsäcke an jedem der beiden Därme deutlich kenntlich, hatten sich letztere typisch in ihre drei Abschnitte gegliedert, und hatte das eine Theilprodukt sogar dentliche Ansätze der Bipinnarienwimperschnur erhalten (Fig. $7 \mathrm{c}$ ). Man wolle diese Vorgänge für die Lektüre der folgenden Abschnitte im Gedächtnis behalten.

Wir kehren nunmehr kurz za unseren Larven mit einem seitlichen Darm zurïck; wie gesagt stützt das eben Geschilderte, wic wir denken, unsere Auffassung desselben als eines »Theil «-Darmes.

1) »Amphioxus.« Journ. of Morph. VIII. 
Zwei solcher Objekte starben, ohne sich viel weiter entwickelt, namentlich ohne Mesenchym gebildet zu haben; in vier anderen Fillen dagegen fand Mesenchymbildung und zugleich ein völliger Ausgleich der Gesammtform statt, so dass der Darm endlich symmetrisch im Ganzen lag und man der Larve fast in nichts mehr ihre Entstehung ansah (Fig. 5 b).

Wirft dieser Ausgleich nun nicht unsere ganze Auffassung des seitlichen Darmes als des Darmes einer Theillarve, eines neuen kleinen Ganzen, in deren Partner der Darm nicht gebildet ist, über den Haufen? Ich halte die Stïtze, welche diese Auffassung darin findet, dass sie allein für die seitliche Darmlage einen zureichenden Grund bietet, fuir zu fest, um gleich so zu schließen; auch denke ich, sind wir aus Theil I im Besitz einiger Mittel zum Verständnis dieses seltsamen regnlatorischen Verhaltens. Wir lernten dort nämlich, dass im Stadium der Mesenchymbildung der osmotische Druck im Blastocöl der Asteriaslarven eine plötzliche starke Steigernng erfährt, welche alle Ektodermdeformationen ausgleicht: wun, eben diese Steigerung des Druckes ziehen wir auch hier heran; auch bei unseren Larven mit seitlich gelegenem Darm fand ja der Formausgleich erst zur Zeit der Mesenchymbildung statt.

Freilich schließt nun dieser ganze Annahmekomplex gewisse Konsequenzen ein, welche Manchem vielleicht bedenklich erscheinen, aber, wie ich denke, doch nicht seltsamer sind als das Geschehen, welches diese ganze Diskussion veranlasste.

Wir schoben die seitliche Lage des Darmes darauf, dass Kraft der zur Zeit der Darmanlage noch nicht ausgeglichenen Konfiguration des Keimes sich die Substanz desselben in zwei Ganze nmorganisirt labe, in deren einem allerdings nur Darmbildnng stattfand; num soll umgekehrt mit eintretender, durch deu osmotischen Druck vermittelter Formregulation des Ektoderms auch wieder Rückumorganisirung zu ein em Ganzen stattfinden : ein »Theildarm《 wïrde also Darm eines Großganzen werden; es wäre eine zweimalige Umorganisirung, ein Schwanken zwischen zwei Kleinganzen und einem Großganzen anzunehmen. Findet man das seltsam, so bedenke man zunächst, dass die für die Massentheilchen des Echinodermeneies anzunehmende Ordnung und damit auch die Vorgänge ihrer Umformung ziemlich einfache Dinge sein werden, sonst würden sie wohl nicht z. B. an jedem beliebigen Keimbruchtheil so prompt und leicht ablaufen ${ }^{1}$ ), sodann

y »0rganisat. d. Eies.s A. E. M. IV. pag. 77, 5 I, $\$ 8$ und 100. 
aber erwäge man Folgendes: vergleicht man die drei Fig. $7 a-7 c$, welche drei Entwickelungsstadien unseres, seltsamen Zwillings darstellen, so findet man, dass auch hier von den zwei vorhandenen Ektodermbuchten mit Auftreten des Mesenchyms die eine ganz, die andere fast verschwand: beide Dairme liegen in einem durchans einheitlichen Raum; ja man fiudet noch mehr: auf Fig. $7 c$ hat zwar jedes Theilindividum zwei Cölome, aber nur eines hat die typische Bipinnarienwimperschnur. Kanu man hieraus nicht sehließen, dass wenigstens das Ektoderm auch bei unserein Zwilling schließlich wieder ein Gauzes geworden ist, während freilich jeder Darm, der ja nun einmal da ist, sich für sich als Kleinganzes ausbildet? Es wiire von groker Bedeutnng diese Fragen und rerwandte Probleme einmal genau zu priifen. Mir war das namentlich desshalb unmöglich, weil das einzige Objekt des gesehildertcn Typus in der letzten Versuchsscrie (19. Miirz) erhalten wurde, welehe ich der Reifeverhältmisse des Asterias wegen anstellen komnte.

Um zum Schluss unsere Auffassung der Larven mit seitlichem Darm bei Echinus und Asterias nochals seharf zu kontrastiren, so denken wir also Folgendes

Bei Echinns:

1) blieb das vom Ei überkommene Großganze der Organisation erhalten bis zur Anlage von Mesenchym and Darm, diese beiden werden daher einheitlich, wenn schon etwas unsymmetrisch angelegt;

2) trat damn nach Anlage von Mesenchym und Darm bisweileu, wemn nämlich der Blastocöldruck zur Wiederherstellung normaler Umrisskonfiguration nicht.genitgte, eine Umorganisation wenigstens des Ektoderms in zwei Kleinganze ein; die Ordnung des Mesenchyms und die Skeletbildung geschahen dam doppelt, im Falle genügenden Blastocöldrueks natiirlich einfach.

Bei Asterias:

1) blieb das vom Ei überkommene Großganze der Organisation nicht bis zur Anlage des Darmes erhalten;

2) trat vielmehr vor dieser eine Umorganisation in zwei Kleinganze ein, wodurch zwiefache Anlage des Darmes bedingt werden konnte, während in anderen Fällen das eine der zwei Kleinganzen noch nicht in für Inscenirung der Darmbildung genügender Weise organisirt war;

3) trat endlich zur Zeit der normalen Steigerung des Blastocöldruckes ein Ausgleich der Umrisskoufiguration und als Folge 
Primäre 11. sekundäre Regulationen in der Entwickelung der Echinodermen. 263

dieser eine Zurückkonstruirung der zwei Kleinganzen zu einem Großganzen, wenigstens in Hinsicht des Ektoderms auf, die Ausbildung des Ektoderms geschah daher einfach.

Echinus und Asterias stehen also nach unserer Meinung in Hiusicht der Cansalverhältnisse ihrer durch kïnstliche Bedingungen erhaltenen Larven mit seitlicher Darmlage in einem wesentlichen Gegensatz, den folgende Tabelle ausdriickt:

\begin{tabular}{c|c|c}
\hline & Echinus & Asterias \\
\hline Anlage des Darmes: & $\begin{array}{c}\text { bestimmt durch das primüre } \\
\text { Großganze, das ein wenig } \\
\text { deformirt ist. }\end{array}$ & $\begin{array}{c}\text { bestimmt durch zwei se- } \\
\text { kundüre Kleinganze. }\end{array}$ \\
Ausbildung der ekto- & $\begin{array}{c}\text { bestimmt durch zwei sekun- } \\
\text { diire Kleinganze oder, bei } \\
\text { genügendem Blastocöldruck, } \\
\text { normaler Weise durch das } \\
\text { erhaltene primäre Groß- } \\
\text { ganze. }\end{array}$ & bestimmt durch ein ter- \\
tiäres Großganze.
\end{tabular}

Vergessen wir nicht, dass anch hinsichtlich der Reihenfolge von Darm- and Mesenchymbildnng Echiniden und Asteriden in einem wesentlichen Gegensatz stehen. -

Wir haben die im Theil I geschilderten Formregulationen den primären ontogenetischen Selbstregulationen zugerechnet und ich denke, wir können das Gleiche mit dem bisher im Theil II (A und Ba) unserer Studie geschilderten Entwickelungsgeschehen thun, denn einmal spielt ja wieder der Faktor des Blastocöldruckes bei ihm eine Rolle, die Grundlage aber für die Umorganisation eines Grobganzen in zwei Kleinganze und umgekehrt ist wohl in polaren Beziehungen der Massentheile der Keimesmaterie gegeben: die Umorganisationen werden durch Faktoren verrichtet, welche, wie ich mich frither $\left.{ }^{1}\right)$ schon ausdrüekte, im Deskriptiv-Normalen die gegebene Organisation der Eier erhalten; für die Organbildungsvorgänge innerhalb des Kleinganzen endlich sind wir auch nicht genöthigt andere als die Faktoren der deskriptiv-normalen Ontogenese einzuführen.

Ein prineipiell der normalen Ontogenese fremder Vorgang, etwa ein Einfluss des bei unserem Zwilling Fig. $7 c$ anzunehmenden neuen tertiären Großganzen auf die in Abhängigkeit von den zwei sekun-

1) »Takt. Reizb.« A. E.EM. III. pag!379. 
dären Kleinganzen gebildeten und nun zum neuen Großganzen nicht passenden Organe (Darmsystem), war nicht zu konstatiren.

Die ersten Anstöße zur Umorganisation der "Ganzen " endlich sind überhaupt keine ontogenetischen Faktoren, sondern sind durch Äußeres, durch den Versuch, gesetzt.

\section{b. Sekundäre Regulationen.}

Wir haben im vorigen Abschnitt gesehen, wie Larren, welche zu einer gewissen Zeit, und zwar ror Beginn jeder Differenzirung; zwei Kleinganze repräsentirten, sich anch in Hinsicht eines Organsystems, des Entoderms, doppelt entwickelten, in Hinsicht anderer, der ektodermalen Organe, aber nicht, da, ehe die Ausgestaltung des Ektoderms begann, die Larve schon wieder, kraft primär-regulirender Faktoren, ein Großganzes darstellte.

Jetzt gehen wir dazu über Objekte zu schildern, welche zu Beginm der Differenzirungen theilweise ein Grofganzes, theilweise aber zwei Kleinganze in örticher Trennung repräsentirten, wobei es sich traf, dass gerade in die Zone der zwei Kleinganzen die ersten Differenzirungsrorgänge fielen und deutlich vou ihnen beeinflusst wurden.

Wenn man sagen wollte, eine Larve wie die der Fig. 3 a repriisentire in ihrem animalen Theil cigentlich zwei Kleinganze, so wirre das riemlich bedentungslos: wir sehen das jedenfalls nicht, können es höchstens vermuthen, denn zu der Zeit, wo die als zwei Kleinganze in Anspruch genommene Larkenzone sich differenzirt, ist ja alle Deformation an ihr ausgeglichen und ist sie jedenfalls (ob non »wieder" oder nicht) ein Großganzes; eben das sehen wir an ihrer Differenzirungsart.

Ich habe nun aber eine ganze Reihe von Larven zu Gesicht bekommen, wo solche Diskussion keine müßige Sache ist.

Fig. 8 stellt eine deformirte Asteriasblastula im Umriss dar, welche etwa von Fig., 2 nur in Nebensächlichem äußerlich versehieden ist; Fig. 2 nun, wie bekannt, erhielt einen Darm und eine sich später ansgleichende Ektodermbucht, Fig. 8 aber erhielt keine Elitodermbucht, dagegen zwei typische neben einander verlaufende Därme (Fig. 9, 10a), welche durch einen mäßigen Zwischenranm von einander getrennt waren.

Wir können nicht anders als annehmen, hier. habe die Deformation den regetativen Theil des Kleinganzen betroffen, und eben in der Deformation seien die Bedingungen zur Umorganisirung seiner 
Theilchen zu zwei neuen Kleinganzen gegeben gewesen, welche Umorganisation sich dann in den an diesem Orte auftretenden Differenzirungen deutlich zeigt.

Es sind im Ganzen fünf Objekte, an denen zwei Därme, welche neben einander, in ihrem ganzen Verlauf getrennt, einherliefen, beobachtet wurden; schildern wir zunächst ihren Entwicklungsgang.

Am Tage nach dem soeben geschilderten (Fig. 10a) Stadium war die Gastrulation vollkommen beendet, beide Därme waren zugieich einander wesentlich näher geriickt (Fig. 10 b), was wohl durch die Konfigurationsänderungen des Ganzen bewirkt war; am folgenden Tage besaß jeder Darm an seinem Ende die typische Blase und hatte Mesenchym gebildet (Fig. 10c). Es entstand nun die Frage: wie viel Cölome wird unsere Larve bilden? Anstatt aber Cölome zu bilden, verschmolzen zunächst die beiden Därme rom Ansatz nach dem freien Ende zu (Fig. 11 a), so dass ein dicker Darm mit ovalem Querschnitt resultirte, der auch nur eine große Endblase besaß.

Es war also eine Larve entstanden, die nur in Kleinigkeiten von einer normalen verschieden war; am nächsten Tage besaß-sie zwei Cölomzipfel und es resultirte endlich eine ganz typische Bipinnarie mit typischer Darmgliederung und zwei Cölomsäcken, deren einer durch den Rückenporus nach außen mündete (Fig. 11 b).

Alle Diskussion auf den Schluss versparend, erwähnen wir zunächst eines Gebildes, welches, dem Ektoderm nach ganz, drei lange Därme neben einander verlaufend anfwies (Fig. 12); die drei Därme bildeten jeder seine Blase und Mesenchym, aber dann degenerirte das Objekt leider und starb. Es sei gleich hier erledigend mitgetheilt, dașs ich die näheren Umstände, welche die Bildung der drei Därme zur Folge batten, anzugeben nicht im Stande bin.

Wir schildern nunmehr eine Kategorie von Larven, welche nicht geringeres Interesse als die mit zwei neben einander verlaufenden Därmen versehenen Individuen darbieten: Diese Objekte begannen die Gastrulation, als ob sie zwei getrennte Därme liefern wollten, nur dass die beiden ersten Darmansätze in wesentlich näherem Kontakt waren, was ein Blick auf Fig. 13 und $14 a$ und ein Vergleich derselben mit Fig. 9 zeigt; im weiteren Verlanf gastrulirten sie jedoch einfach, so dass die vollendete Gastrula einen an seinem freien Ende gespaltenen Darm besaß (Fig. 14b). Bisweilen glich sich die Gabelung ans und resultirte eine Mesenchymlarve mit einer Endblase (Fig. 14c), die sich dann ganz normal weiter entwickelte, bisweilen 
war die Mesenchymblase wenigstens schwach gespalten (Fig. 15) und in mehreren Fällen (Fig. 16,17a) endlich war die Gabelung des Endes dentlich auf zwei kommunicirende, Mesenchym bildende Blasen übergegangen.

Wie viel Cölomzipfel und weiterhin Cölomsäcke ließen sich nun wohl ron so einer Larve erwarten? Besaß sie doch zwei. getrennte Darmenden und wissen wir doch, dass Därme von Theillarven je zwei Cölome zu bilden vermögen (Fig. 7 c). Unsere Objekte bildeten aber, wie in jedem Fall mit besonderer Sorgfalt konstatirt ward, im Ganzen nur zwei Cölome (Fig. 17b) und zwar ging die aus der ursprünglichen Gabelung des Darmendes überkommene Zipfligkeit, der Endblase ohne Weiteres in die Cölomzipfel über; das überkommene Geschehnis ward hier, wie es vorlag, benutzt.

In solchen Larven lag nach Bildung ihrer zwei Cölome nun, abgesehen ron etwaigen kleinen Verzerrungen des Ektoderms, nichts vor, was sie irgendwie von einer normal entstandenen Larve hätte unterscheiden können; die aus jedem Objokte herrorgegangene Bipinnarie ward mit besonderer Sorgfalt, nach Abtödtung mit Formol, studirt: stets gab es zwei Cölomsïcke, deren einer nach außen mündete, niemals vier; die Darmgliederung sowie die ganze änßere Formgestaltung waren durchaus normal (Fig. 18).

Ziehen wir nunmehr einige Schlüsse aus den seltsamen vorstehend mitgetheilten Thatsachen:

Was zunächst die erste Entstehung der echten Doppeldärme (Fig. 9) anlangt, so haben wir bereits oben betont, dass sie uns ihren Grund in einer Umorganisirung des vegetativen Larventheils zu zwei Kleinganzen ihren Grund zu haben scheint, und es ist wohl in der That schwer diese Auffassung abzulohnen. Ist num eine ähnliche Auffassung auch für diejenige Darmbildungsart zulässig, bei welcher der Darm seine Entstehung doppelt beginnt (Fig. 13) aber cinfach endigt, so dass ein an seinem freien Ende gabelig. gespaltener Darm resultirt (Fig. 14b)? Erinnern wir uns, um diese Frage zu prïfen, einmal der Bildungsweise des Echinodermendarmes unter normalen Umständen: wie wir durch MoRGAN1) wissen, ist seine erste Andeutung eine an einem Pol gelegene dicht-zellige Platte, deren Entstehungsart uns hier nicht weiter interessirt; indem die Zellen dieser Platte, welche bis dahin nicht gewachsen waren,

1) »Partial Larvae.» A. E. M. II. pag. $84 / 5$. 
plötzlich durch Wasseraufnahme eine starke Streckung erfahren, wird die Platte, da ihr nach den Seiten das Ektoderm Widerstand entgegensetzt, zu einem langen Schlauche, d. i. eben zum Urdarm. Das ist wenigstens in seinen gröbsten Zügen die Genese dieses Organs; wenden wir das, was sie uns lehrt, auf die Entstehung echter Doppeldärme an, so müssen wir diese also von der Ausbildung. zweier getrennter Platten herleiten: diese beide Platten sind die nächsten 'Folgen der Ausbildung' zweier Kleinganzer. Für unsere Auffassung spricht offenbar auch die Thatsache, dass die beiden Därme Anfangs deutlich durch einen Zwischenraum von einander getrennt sind (Fig. 9, 10a) und erst später, wohl durch die Configurationsänderungen des Ganzen, in ummittelbaren Kontakt kommen (Fig. $10 c$ ), worauf sie dann verschmelzen.

Können wir nun die Anlage zweier Platten anch für die erste Entstehung unserer Gabeldärme postuliren? Tch denke nicht, und zwar desshalb nicht, weil doch die Einheitlichkeit des Weiterwachsens dieser Gebilde zeigt, dass hier das Entodermmaterial kontinuirlich ron Anfang an in einander iibergeht, also nicht gut von zwei getrennten Platten ausgegangen sein kann; ich möchte also annehmen, dass die Gabeldärme. doch, dem Anschein vielleicht zum Trotz, Gebilde eines Großganzen sind, die nur desshalb, weil die Darmplatte, von der sie ausgingen an den Deformationsort fiel und daher selbst deformirt wurde, eine Abweichung vom normalen Formverhalten darbieten: wenn man nur anzunehmen geneigt ist, dass eine so deformirte Platte etwa an zwei seitlichen Stellen ihre zum Schlanche führende Streckung etwas eher beginnt als im Centrum, müchte man schon die anfängliche Schein-Doppelnatur und die spätere Endgabelung ihrer Descendenten ableiten können; man ersieht. iibrigens an Fig. 13, welche den Anfang eines Gabeldarmes repräsentirt, dass hier, im Gegensatz zu Fig. 9, von Anfang an Kontakt des entodermalen Materials besteht.

Um im Verlaufe der weiteren Diskussion zunächst bei unseren Objekten mit Gabeldarm zu verweilen, so erscheint als das seltsamste Faktum in ihrer Entwickehung die Cölombildung: bei solchen Larven, deren Gabelung sich nicht ansglich, sondern erhalten blieb, lieferte jeder Gabelast eine Mesenchymblase aber an ch nur einen Cölomzipfel und Cölomsack, in den er eben, wie er da war, überging. Auffallend ist das, denke ich, aus folgenden Gründen: sehen wir anch den Gabeldarm in seiner ersten Entstehung nicht eigentlich als Doppelbildung, als Folge zweier Kleinganzer, an, so ist 
doch mit seiner Trennung vom anderen und mit seiner Selbständigkeit im weiteren Verlanf jedes Gabelende nachtrïglich zu einem Kleinganzen geworden: es sollte also zwei Cölome bilden. Dass es nur ein Cölom bildet, sclieint mir hier das Inkrafttreten einer neuen, der normalen Ontogenese fremden Geschehensart anzadeuten, kurz es scheint mir in der Bildung nur eines Cöloms an einern Organ, von dem man nach unseren sonst erworbenen Kenntnissen zwei Cölome erwarten sollte, ein Fall seknndärer ontogenetischer Regulation vorzuliegen. Gerade so wie bei Regenerationeu ein Organ, da das Canze es erheischt, mehr bildet als es im Normalen thut, so bildet es hier, da das Ganze es erheischt, weniger, eine Parallele, welche die von mir stets befuirwortete $\left.{ }^{1}\right)$ Trennung des Regenerationsbegriffs yom Begriff der Regrtlation einmal wieder in giinstiges licht setzt.

Wenn wir nunmehr von unseren Doppeldarmlarven reden wollen, so haben wir hier natürlich einen zweiten Fall sekundärer Regulation anfs aller handgreiflichste vor Augen, und zwar in der Verschmelzung der beiden Därme zu cinem einheitlichen Darm; auch das ist ein der normalen Ontogenese fremder Vorgang, geschehen im Dienste des Ganzen, welches eben ein einheitliches Grobganze ist.

Die zweite sekundäre Regulation an Larven mit Doppeldarm ist der an den Gabeldarmlarven geschilderten verwandt; es ist die Bildung nur im Ganzeu zweier Cölome, wo doch zwei Mesenchymblasen, also die Vorbedingungen zweimal zweier Cölome vorhanden gewesen sind; auch hier geschieht, was das Ganze zu harmonischer Ausbildung erheischt, das Resultat ist denn auch eine Bipinnaria, der man ihre Entstehungsart nicht ansieht ${ }^{2}$ ) (Fig. 11b).

1) z. B. »Organzellen.« A. E. M. II. pag. $188 \mathrm{ff}$.

2) Wenn man ihre Darmzellen zählen und das Resultat mit der Zahl der Darmzellen normaler Bipinnarien vergleichen wollte, wiirde man doch vielleicht einen kleinen Unterschied konstatiren. MORGAN (A. E. M. II.) konstatirte für Echiniden, dass der Darm von aus einer der ersten Blastomeren gezogenen Gastrulae die normale, also eine relativ zu hohe Zellenzahl besitzt; das trifft nun für Asterias nicht zu, wie in einer folgenden Studie mitgetheilt werden wird; hier ist die Darmzellenzahl von Theillarven ungefähr halb so groß wie in Ganzlarven, also proportional ungefähr richtig, aber ganz genau halb ist sie doch nicht und so mag denn auch in einem Darm, der aus zwei verschmolzenen Theildärmen entstand, die Zellenzahl nicht ganz normal sein. 
Vergleichen wir zum Schlnss einmal unsere Doppeldarmlarven mit jener oben gesehilderten und in Fig. $7 c$ abgebildeten Larve, die in Bezug anf alles Entodermale wenigstens, ein echter Zwilling war. Wesshalb hier zweimal zwei Cölome, zwei dreigliedrige Därme und bei unseren jetzt geschilderten Objekten (Fig. 1Ib) nur im Ganzen zwei Cölome und ein Darm? Der nächste Grund des verschiedenen Verhaltens liegt offenbar in einer Zufälligkeit individuellen Charakters, nämlich in der verschiedenen Art der Deformation, welche die Operation erzengte; diese Verschiedenheit bestimmte die verschiedene Lage der Theildärme, bestimmte es, dass sie eimmal ziemlich nahe bei einander (Fig. 10 $\boldsymbol{c}$ ), das andere Mal weit von cinander entfernt (Fig. $7 a$ ) verliefen, damit war wieder das eine Mal die Möglichkeit der sekundären Regulation der Verschmelzung gegeben, das andere Mal nicht, und von dem Eintreten oder Fehlen dieser hing wieder die Zahl der entstehenden Cölome kraft einer zweiten Sekundärregulation ab. Wir können sagen: wären die Dürme unserer Doppeldarmlarven weiter von einander gelegen gewesen, so hätten sie sich jeder als Kleinganzes vollständig mit je zwei Cölomen differenzirt. Von Zufälligkeiten hängt also die Auslösung der sekundären Regulationen ab, wiewohl natïrlich sie selbst in ihrem Ablauf durchaus geordnete Geschehensarten sind, von Zufälligkeiten, die, da doch alle Eier gleichzeitig ein und demselben Versuch unterworfen wurden, entweder in ihrem individuellen Rieaktionsvermögen gegenüber dem Schüttelverfahren oder in ihrer gerade mehr oder weniger gesehützten Lage im Eihaufen, wahrscheinlich wohl in ersteren, gelegen sind 1 ).

Hat eine Larve, die im ektodermalen Theil einheitlich ist, zwei Dürme gebildei und diese liegen so, dass sie zur Berührung gelangen können, so kann kraft sekundärer Regulationen ein durchaus einheitliches Ganze noch geschaffen werden; hat sie dagegen zwei von einander weit entfernt gelegene Därme gebildet, so vermag alle Einheitlichkeit des Ektoderms den einen der Därme nicht

1) Vgl. „Über den Antheil zufalliger individueller Verschiedenheiten an on togenetischen Versuchsresultaten.« A. E. M. III, pag. $295 \mathrm{ff.}$ Oben sprach ich es nicht mit voller Sicherheit ans, dass Verschiedenheiten im Entwickelungsgebahren meiner Versuchsobjekte auf individuelle Verschiedenheiten zurückzuführen seien, in anderen Fällen lässt sich das aber absolut bestimmt behaupten, so z. B. wenn in eben demselben Gefäß mit verdünntem Seewasser die einen Eier sich als ein Granzes, die anderen sich als zwei Ganze oder in Übergangsformen entwickeln. 
wegzuschaffen. Was lernen wir daraus? Tch denke, wir sehen hier, dass sekundäre Regulationen, in gewissem Sinne wenigstens, als Specialgeschehuisse oder Specialeinichtungen, aber nicht ohne Weiteres als Aushiüsse einer allgemeinen vitalen Gesetzlichkeit aufzufassen sind, was ja anch die so große Verschiedenheit der Regenerationsfühigkeit sowohl hinsichtlich versehiedener Formen als auch hinsichtlich verschiedener Organe bei derselben Form lehrt.

Kommt nuu dieser Schluss der »Maschinentheorie des Lebens « 1) zil Gute ${ }^{2}$ ) oder nicht?

Napoli, den 1. Juni 1896.

\section{Erklärung der Abbildungen.}

Tafel VIII.

Bis auf die Figuren 1, 2, 4, $7 e, 10 a, 11 b, 13,18$, II, VII b, VIII $e$, sind alle Abbildungen Skizzen, die ohne Camera nach Besichtigung der frei schwimmenden Larve rasch entworfen wurden; sie sind genau daher nur in Bezug anf solche Verhiltrisse, die den eigentliehen Gegenstand des Studiums bildeten (Ektodermbuchten, Lage des Mesenchym-Darmbildungsortes bei Echiuus, Darmlage).

Asterias arabische Ziffern!.

Fig. 1. Blastnla mit zwei einander gegenüber liegenden Buchten. Zxrss Apochr. $16 \mathrm{~mm}$. Oc. 4.

Fig. 2. Blastula mit einer Bucht. Zerss wie 1.

Fig. 3a. Gastrula mit starker Ektodermbucht.

Fig. 37. Dieselbe Larre im Beginn der Mesenchymbildung, Beginn des Fktodermausgleichs.

Fig. 3e. Dieselbe Larve, Ausgleich vollendet.

Fig. 4. Gastrula mit seitlich gelegenem Darm. Zasss wie 1.

Fig. 5 a. Gastrula wie 4, der Darm liegt ganz deutlich in einer der beiden durch die Ektodermeinschnürungen bestimmten Blasen und entspringt anch in ihr.

Fig. 5b. Dieselbe Larve im Beginn der Mesenchymbildung; Formansgleich fast vollendet.

Fig. 6. Gastrula mit zwei woit von einander entspringenden und liegenden Därmen; in jeder Blase liegt einer.

1) Biolog. Centralbl. XVI. pag. $353 \mathrm{ff}$.

$\Rightarrow$ Roux neigt bekanntlich dazn, in der Beschrïnktheit der Regulationsvorgïnge eine Stiitze fuir das zu sehen, was ich ibre Maschinennatur nenne, WOLFF nicht; ich denke, diese Beschränktheit ist weder pro noch contra zu verwerthen. 
Primäre a. sekundire Regulationen in der Entwickelung der Echinodermen. 271

Fig. 7a. Gastrula mit zwei weit von einander entfernt liegenden, ungleichsinnig orientirten Därmen.

Fig. $7 b$. Dieselbe Larve nach vollendeter Mesenchymbildung, der eine Darm bereits in Cölombildung, die eine Ektodermbucht im Ausgleich.

Fig. $7 c$. Dieselbe Larve einen 'Tag spiiter, jeder Darm hat zwei Cölomsücke gebildet, um den einen Darm als Centrum hat sich die Bipinnarienwimperschnur angelegt, die eine Ektodermbucht ist ganz ausgeglichen. Das Ektoderm dieser Larve ist vielleicht als ein Ganzes anzusehen, siehe Text. Zerss wie 1.

Fig. 8. Blastula mit einer Ektodermbueht, welche, wie die spütere Ausbildnng zweier ziemlich nahe bei einander gelegenen Därme zeigte, am vegetativen Pol sich befand.

Fig. 9. Gastrula mit zwei noch kleinen Därmen, die später einander näher rückten und endlich verwuchsen.

Fig. $10 \mathrm{a}$. Gastrula mit zwei nahezn vollendeten, einander ziemlich nahe liegenden Dürmen Zkiss wie 1.

Fig. 107. Dieselbe Larve, die Därme ansgewachsen und einander nahe geriickt.

Fig. 10c. Dieselbe Larve nach vollendeter Mesenchymbildnng. Es sind zwei Mesenchymblasen vorhanden, die Därme liegen einander sehr nah, sie kamen darauf zur Berührung und verschmolzen; es resultirte eine typische Bipinnaria.

Fig. 11 a. Ähnliches Individuun (roh skizzirt) im Stadium der halb ver: schmolzenen Därme (die Figuren $10 a-$ e künnten auch für die früheren Stadien dieses gelten!

Fig. 11\%. Die völlig normal gestaltete Bipinnaria, welche aus ihm resultirte. ZeIss wie 1.

Fig. 12. Gastrula mit drei neben einander verlaufenden Däirmen.

Fig. 13. Larve im Beginn der Gabeldarmbildung. Zwiss wie 1.

Fig. 14a. Ähnliche Larve, zugleich mit Ektodermbucht.

Fig. 14b. Dieselbe Larve mit vollendetem Gabeldarm, Ektodermbucht ansgeglichen.

Fig. I4c. Dieselbe Larve nach vollendeter Mesenchymbildung. Gabelung verschwunden, eine Endblase.

Fig. 15. Mesenchymgastjula mit gespaltener Endblase, aus einer Gastrula mit Gabeldarm hervorgegangen.

Fig. 16. Mesenchymgastrula mit zwei Endblasen, ans einer Gastrula mit Gabeldarm unter direkter Benutzung der Gabelenden hervorgegangen.

Fig. $17 a$. Mesenchymgastrula wie die vorige, noch mit Ektodermbucht.

Fig. 17b. Die Gabelènden sind zur Cölomzipfelbildung verwandt; Ektodermbucht ausgeglichen.

Fig. 18. Völlig normal aussehende Bipinnaria, aus einer Larve wie Fig. 10 hervorgegangen. ZEIss wie 1.

\section{Echinus (römische Ziffern).}

Fig. I. Zerrblastula mit einheitlichem, symmetrischem Mesenchymbildungsort in der Ringbucht; von der Seite.

Fig. II. Zerrblastula vom animalen Pol, das Mesenchym (ca. 60 Zellen) ist einheitlich, ringförmig, bilateral geordnet. Man sieht die Darmplatte (schraffirt). ZEISS D* Oc. 2. 
Fig. III. Gastrula, aus einer Zerrblastula hervorgegangen, Bucht des Ektoderms fast ansgeglichen.

Fig. IV. Prismengastrula aus einer Zerrblastula hervorgegangen, völlig normal.

Fig. Va. Gastrula mit sehr seitlich verlaufendem Darm, aus einer Zerrblastula mit nicht ganz symmetrischer Darmplatte (wie VIII $t$ ) hervorgegangen.

Fig. Vb. Dieselbe Larve im Beginn der Prismenform, ausgeglichen.

Fig. VI. Prismengastrula, ans einer Zerrblastula hervorgegangen, deren Darm auffallend spät noch der gewïlbten Fläche anliegt, so dass er mit dem bereits vorhandenen Mund nicht kommnnicirt; er sollte liegen, wie die gestriehelten Linien angeben. Später vollzog übrigens der Darm die normale Wendung und es resultirte ein normaler Pluteus.

Fig. VII $a$. Gastrula mit seitlichem Darm (ähnlich Va).

Fig. VIIb. Dieselbe Larve als Pluteus; Skelet, Mnndfeld, Mund normal, der (normal-dreigliedrige) Darm liegt aber um $90^{\circ}$ falsch $(\omega=$ Darmansatz = Blastopor $=$ After), so dass er mit dem Mund nicht kommunicirt. ZEISs $D^{*}$ Oc. 4 .

Fig. VIII $a$. Zerrblastnla mit asymmetrisch gelegenem Mesenchym-Darmbildungsort.

Fig. VIII $b$. Dieselbe Larve als Gastrula mit seitlichem Darm.

Fig. VIII $c$. Dieselbe Larve; im Gegensatz zu $V a$ und $b$ unterblieb der Formansgleich im Ektoderm, dieses wurde daher zu zwei Kleinganzen umorganisirt. Man sieht zweimal zwei Skeletanlagen, zwei Wimperschnuranlagen, aber nur einen dreigliedrigen Darm. Die äußere Plateusform ist am besten an demjenigen Kleinganzen ausgebildet, in dem der Darm nicht liegt. Zeiss $D^{*}$ 0c. 2.

Fig. IX. Zerrlarve, deren Mesenchym sich, den beiden Ektodermblasen ( $>$ Kleinganzen $\leftrightarrow$ entsprechend, um zwei Centren geordnet hatte; das Objekt starb ohne Darmbildung; sehr schematisch.

\section{Inhaltsübersicht.}

Einleitung . . . . . . . . . . . . . . . . 247

I. Der Ausgleich der äußeren Form bei faltigen Echinodermenblastulis . 249

A. Asteriden . . . . . . . . . . . . . . . . . . . 248

B. Echiniden . . . . . . . . . . . . . . 250

If. Die Darmentwickelung von deformirten Echinodermenblastulis und ibre Regulationen. . . . . . . . . . . . . . . . . . . . . 254

A. Echiniden (primäre Regulationen,. . . . . . . . . . . 254

B. Asteriden . . . . . . . . . . . . . . . . . 259

a. Primïre Regulationen .............. . 259

b. Sekundäre Regulationen . . . . . . . . . . 264

Erklärung der Abbildungen . . . . . . . . . . . . . 270 